\title{
La aplicación de los números complejos en la enseñanza de la geometría plana
}

\author{
M.A. Murray-Lasso \\ Unidad de Enseñanza Auxiliada por Computadora \\ Departamento de Ingeniería de Sistemas, División de Estudios de Posgrado \\ Facultad de Ingenieria, UNAM
}

(recibido: enero de 1999; aceptado: agosto de 2000)

Resumen

Los números complejos aplican las mismas leyes aritméticas que los números reales, por lo que son casi tan fáciles de manipular como éstos. Su interpretación geométrica en un plano, como segmentos de recta dirigidos, los hace una herramienta ideal para estudiar geometría plana. Debido a que están definidas, la multiplicación y división de números complejos, además de tener interpretaciones geométricas útiles, el autor considera que son más versátiles que los vectores bidimensionales para los cuales solamente está definida la multiplicación por un escalar y el producto punto, por lo que no hay división.

En este artículo, se tratan brevemente las cuatro operaciones fundamentales de la aritmética de los números complejos y sus interpretaciones geométricas. Con este mínimo herramental se procede a exhibir la solución de varios problemas y la demostración de un par de teoremas que serían difíciles de resolver por medio de técnicas convencionales de la geometría (es decir, detección de ángulos iguales, de figuras semejantes, la aplicación del teorema de Pitágoras, etc.) Una ventaja adicional del uso de números complejos para el estudio de la geometría, es la generalidad de los resultados obtenidos, generalidad que para lograrla con métodos geométricos convencionales requiere el tratamiento de múltiples casos especiales. Lo anterior se ilustra ampliamente en este trabajo.

Descriptores: complejos, geometría, enseñanza, desargues, Von Aulen

\begin{abstract}
The same arithmetic rules are applicable to both complex and real numbers; the former can be therefore as easily manipulated as the latter. Their geometric interpretation in a plane, as oriented segments of a straight line, make them an ideal tool for the study of plane geometry. Because the multiplication and the division of complex numbers are defined, in addition to having available useful geometric interpretations, the author considers these numbers to be more versatile than two-dimensional vectors for which only the multiplication by a real number and the point product are defined and therefore no division is available. This paper summarizes the four fundamental operations of the arithmetic of complex numbers and their geometric interpretations. With these minimal tools the solution to various problems is presented as well as the demonstration of a couple of theorems that would be otherwise difficult to solve by means of conventional geometric techniques (i.e., detection of equal angles, and of similar figures, the application of Pythagoras' theorem, etc.). An additional advantage derived from the use of complex numbers for the study of geometry is the general nature of the results thus obtained because to achieve them through conventional geometric methods it is necessary to deal with multiple special cases. These operations are discussed in detail in the paper.
\end{abstract}

Keywords: complex numbers, geometry, learning processes, desargues, Von Aulen. 
DOI: http://dx.doi.org/10.22201/fi.25940732e.2000.01n5.023

\section{Introducción}

La enseñanza de un tema se enriquece cuando se señalan sus conexiones con otros temas. Uno de los grandes descubrimientos de las matemáticas lo realizó Descartes, cuando señaló que la geometría se puede relacionar íntimamente con el álgebra. Este gran descubrimiento originó la geometría analítica, la cual tuvo una gran influencia en establecer la íntima conexión que tiene la física con las matemáticas, permitiendo entre otras cosas, el estudio analítico del movimiento por medio del cálculo diferencial e integral.

Un tema que el autor considera no está suficientemente enfatizado en la enseñanza de las matemáticas, es la relación existente entre los números complejos y la geometría plana. El número complejo es una posible alternativa a los vectores bidimensionales debido a su interpretación en el plano de Argand. La ley de la suma de los números complejos, en la cual se suman separadamente las partes reales y las partes imaginarias para obtener la parte real e imaginaria de la suma, respectivamente, coincide totalmente con la ley de la suma para vectores bidimensionales, en la cual se suman separadamente los componentes sobre los ejes $x$ e $y$ para obtener los componentes sobre dichos ejes de la suma de dos vectores. Asimismo, tiene una interpretación muy útil en el producto de dos números complejos.

Al igual que la ortogonalidad entre vectores de cualquier número de dimensiones, se detecta por medio del producto punto, producto escalar o producto interno entre vectores, por ello es fácil detectar cuando se usan números complejos. Estas ventajas hacen que muchas demostraciones de geometría plana se logren con más facilidad y generalidad cuando se utilizan los números complejos que con los métodos convencionales de la geometría.

El resto de este artículo se orientará a mostrar algunas ilustraciones de las posibilidades del uso de los números complejos en el estudio de la geometría plana.

En algunas demostraciones posteriores habrá necesidad de representar un segmento de recta. Los números complejos se prestan para hacer dicha representación de una manera muy simple. Sea $P$ un punto del plano complejo por el que pasa una recta y sea $Z$ un número complejo representado por una flecha cuya dirección es la de la recta en cuestión. Entonces una ecuación paramétrica de la recta con la dirección de $Z$ y que pasa por el punto $P$ es

$$
W=P+t Z
$$

donde $t$ es un parámetro que puede tomar cualquier valor real. Una posible interpretación de la ecuación es que, es el lugar geométrico de un punto que parte del punto $P$ y se desplaza con una rapidez en $\mathrm{m} / \mathrm{s}$ igual a la magnitud en metros del número complejo $Z$ sobre la recta que pasa por $P$ y tiene la dirección y sentido del número complejo $Z$ para $t$ $>0$. Si el número $t$ es negativo, el desplazamiento es en sentido contrario (Figura 1).

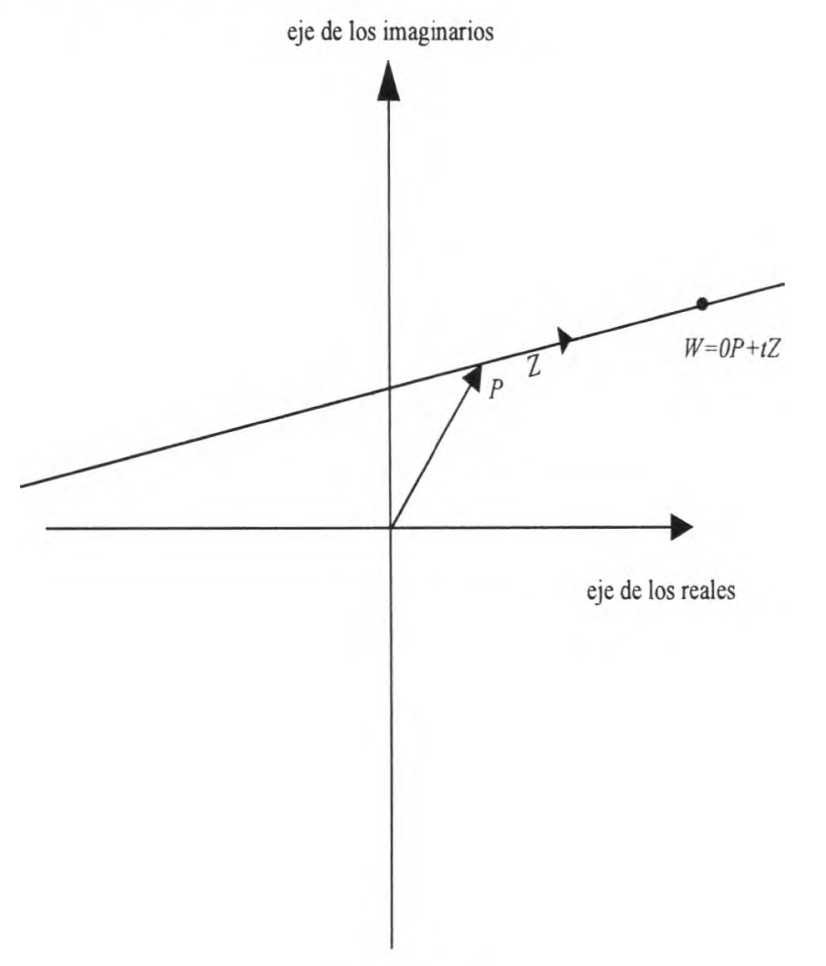

Figura 1

En ocasiones, es necesario encontrar el punto en el que se cruzan dos rectas representadas por una ecuación en números complejos. Ahora se anota un ejemplo específico para ilustrar. Sean las ecuaciones $W_{1}=i+t_{1}(1-i)$ y $W_{2}$ $=2+t_{2}(1+i)$. Se desea encontrar el punto de cruce de las dos rectas. El punto de cruce será el lugar donde $W_{1}=W_{2}$. Esto nos lleva a la ecuación $i+t_{1}(1-i)=2+t_{2}(1+i)$ que equivale a las siguientes dos ecuaciones (separando las partes reales e imaginarias) $t_{1}-t_{2}=2, t_{1}+t_{2}=1$. Esto da como solución $t_{1}=3 / 2, t_{2}=-1 / 2$. Por lo tanto, el punto de cruce es $W_{1}=W_{2}=i+(3 / 2)(1-i)=3 / 2-i / 2$. Geométricamente la primera ecuación pasa por el punto $0+i$ y tiene una pendiente de 45 grados abajo de la horizontal. La segunda recta pasa por el punto $2+0 i$ y tiene una pendiente de 45 grados arriba de la horizontal. El punto de cruce se muestra en la figura 2 y es $3 / 2-i / 2$.

Para representar una circunferencia centrada en el origen del plano complejo que encierra un círculo con radio $|\mathrm{R}|$; se puede utilizar la siguiente ecuación paramétrica

$$
W=|R| e^{i(t+\theta)}, \quad 0 \leq t \leq 2 \pi
$$




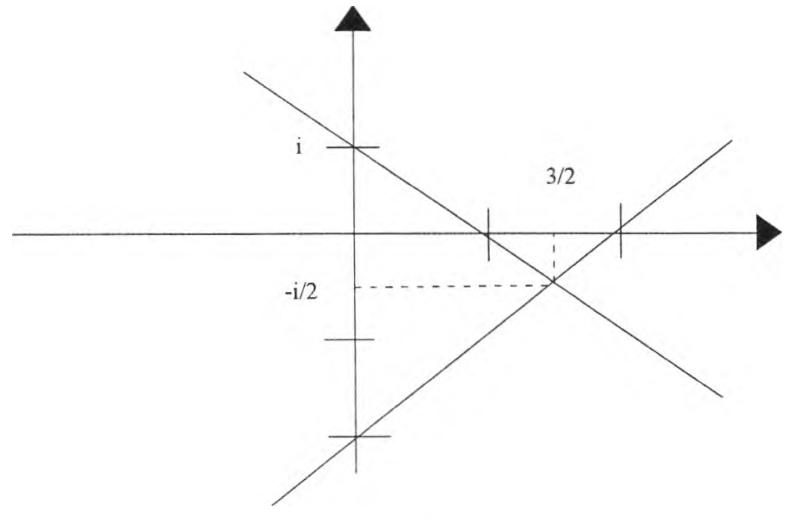

Figura 2

La interpretación de la ecuación paramétrica de la circunferencia es que, es el lugar geométrico de un punto con movimiento circular uniforme a una velocidad angular de un radián/s sobre una circunferencia con centro en el origen del plano complejo y radio $|R|$. En el instante $t=0$, el punto está en el extremo de un radio que forma un ángulo $\theta$ con el eje de los reales. Si se desea que el centro del círculo sea el punto $P$ del plano complejo, entonces la ecuación se convierte en

$$
W=P+|R| e^{i((t+))}, 0 \leq t \leq 2 \pi
$$

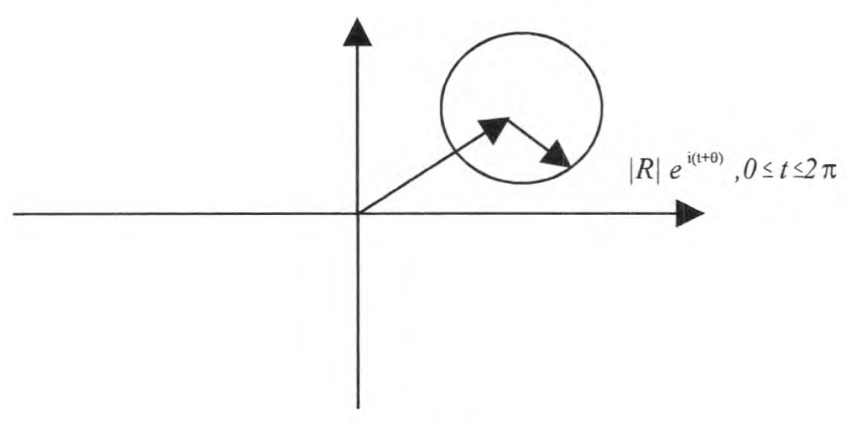

Figura 3

No se pretende, con lo anterior, convertir este artículo en un tutorial de números complejos; por esta razón, el resto de este trabajo se dedica a exhibir algunos ejemplos de posibilidades de aplicación de los números complejos al estudio de la geometría plana.

\section{Solución de un problema geométrico}

El siguiente problema se tomó de un libro de acertijos matemáticos (Gardner, 1981). En la figura 4a se muestran tres cuadrados adyacentes y tres líneas que con la horizontal forman ángulos $\alpha, \beta, \gamma$. El problema es que con los datos de la Fig. 4a hay que demostrar que $\alpha=\beta+\gamma$. La demostración que da como respuesta el autor Gardner, utiliza la Fig. $4 \mathrm{~b}$ argumentando:

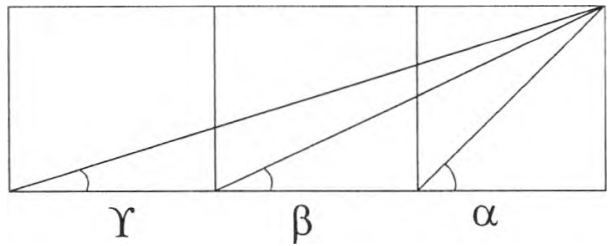

Figura $4 a$

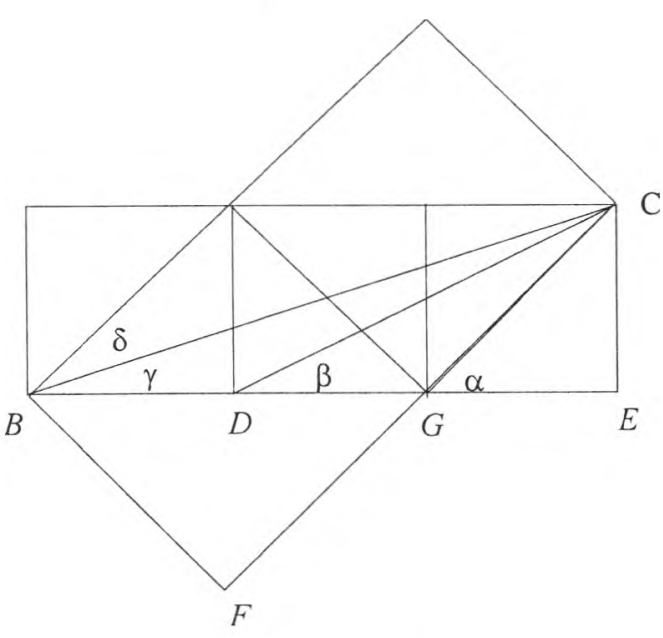

Figura $4 b$

Construya el rectángulo inclinado como se muestra en la Fig. 4b. El triángulo $A B C$ es semejante al triángulo EDC y los ángulos $\delta$ y $\beta$ se corresponden, por lo que son iguales. El ángulo $\alpha$ es igual a $\gamma+\delta$, por correspondientes, ya que las líneas BA y FC son paralelas.

Se tiene entonces $\alpha=\gamma+\beta$ que es lo que se quería demostrar.

Utilizando números complejos, la demostración se basa en que el producto de dos números complejos tiene un ángulo igual a la suma de los ángulos de cada uno de los factores. Los números complejos que representan a los segmentos $G C, D C$ y $B C$, son respectivamente

$$
1+i, 2+i \text { y } 3+i
$$

(hemos supuesto que los cuadrados pequeños tienen lado de longitud unitaria. Multiplicando los dos últimos se tiene $(2+i)(3+i)=5+5 i=5(1+i)$. Como se ve el resultado, tiene un ángulo de 45 grados y por lo tanto tiene la misma pendiente que el segmento de recta GC de la Fig. 4 b. La demostración por medio de números complejos tiene la ventaja de que no es necesario que al estudiante se le ocurra construir el rectángulo auxiliar de la Fig. 4b. Otro método de solución sería utilizar fórmulas trigonométricas capaces de verificar que $\arctan 1 / 3+\arctan 1 / 2=\arctan 1$. Esto tiene la desventaja de que hay que conocer dichas fórmulas, que en general, no es parte del herramental normal que utilizan los no especialistas. 


\section{Demostración del teorema de Von Aubel}

El autor Gardner (1981) hace referencia a un teorema de Von Aubel, en el cual, si se dibuja un cuadrilátero convexo - una figura es convexa si, dados dos puntos cualesquiera que están dentro de la figura, todos los puntos, en el segmento de recta que une a los puntos también están dentro de la ella- y se construyen cuadrados hacia afuera de la figura apoyados sobre los lados del cuadrilátero, como se muestra en la Fig. 5, las líneas que unen a los centros de cuadrados de lados opuestos son perpendiculares y tienen la misma longitud.

Aunque Gardner no da una demostración del teorema de Von Aubel, hace referencia a una demostración vectorial dada por Kelly P. (1966). Aquí se hará una demostración utilizando los números complejos para que el lector aprecie la facilidad con la que se maneja esta herramienta y la generalidad de los resultados que se obtienen. Yaglom (1962) ofrece una demostración utilizando operaciones de rotación y siguiendo la teoría de operaciones invariantes introducida por Klein (1939).

En la Fig. 6 se muestra una construcción equivalente a construir cuadrados sobre los lados del cuadrilátero y encontrar sus centros. Arbitrariamente ponemos el origen del plano complejo coincidiendo con una de las esquinas del cuadrilátero. (Punto O de la Fig. 6)

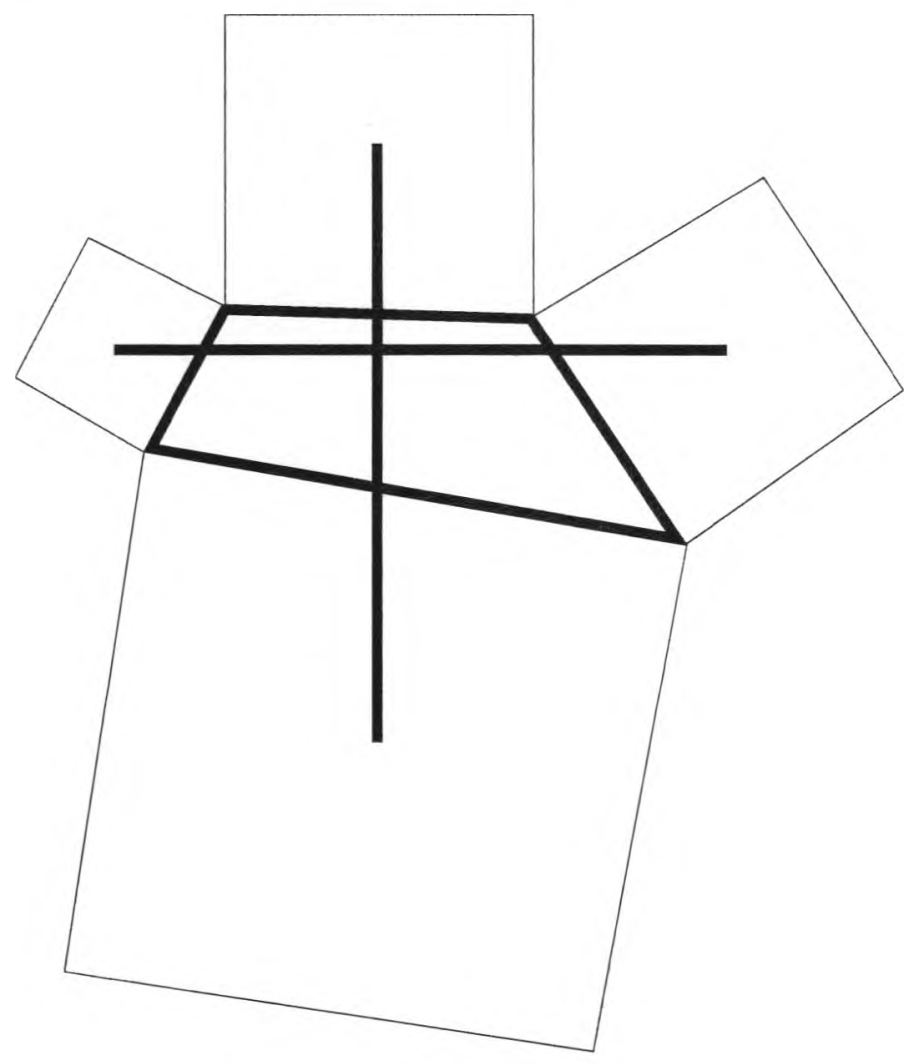

Figura 5

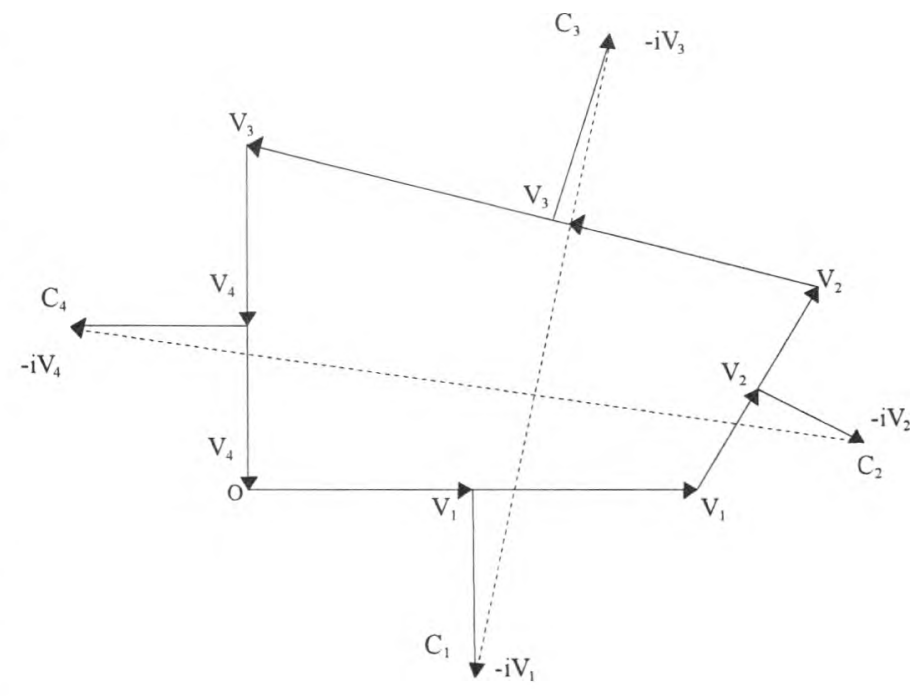

Figura 6

Partiendo del origen, se representan por medio de los números complejos $2 \mathrm{~V}_{1}, 2 \mathrm{~V}_{2}, 2 \mathrm{~V}_{3}, 2 \mathrm{~V}_{4}$, los segmentos de recta orientados con las mismas direcciones y las orientaciones que se muestran en la Fig. 6 de los cuatro lados del cuadrilátero. Al recorrer el cuadrilátero en sentido antihorario se tiene

$$
2 V_{1}+2 V_{2}+2 V_{3}+2 V_{4}=0
$$

o lo que es lo mismo $V_{4}=-V_{1}-V_{2}-V_{3}$,

debido a que el cuadrilátero termina en el origen. Para llegar a los centros de los cuadrados construidos en cada lado del cuadrilátero, a medio lado de cada uno de los lados del cuadrilátero sumamos el número complejo $-i V_{i}$ para $i$ $=1,2,3,4$ como se muestra en la Fig. 6. - Recordar que $-i$ como factor, rota a una flecha a la que multiplica 90 grados en sentido de las manecillas del reloj-. El número complejo $\mathrm{C}_{1}-\mathrm{C}_{3}$ representa a la flecha que va del centro del cuadrado sobre el tercer lado al centro del cuadrado sobre el primer lado. Se puede calcular directamente sobre la gráfica viendo qué flechas hay que recorrer y en qué sentidos para pasar de la cola de $\mathrm{C}_{1}-\mathrm{C}_{3}$ a su punta.

Por lo tanto, podemos escribir por inspección (viajando por la parte derecha de la Fig. 6)

$$
\begin{gathered}
C_{1}-C_{3}=i V_{3}-V_{3}-2 V_{2}-V_{1}-i V_{1}= \\
\left(-V_{1}-2 V_{2}-V_{3}\right)-i\left(V_{1}-V_{3}\right)
\end{gathered}
$$

Haciendo lo mismo para el otro par de centros de cuadrados adyacentes a los otros dos lados opuestos tenemos-viajando por la parte inferior de la figura 6-. 


$$
\mathrm{C}_{2}-\mathrm{C}_{4}=i \mathrm{~V}_{4}+\mathrm{V}_{4}+2 \mathrm{~V}_{1}+\mathrm{V}_{2}-i \mathrm{~V}_{2}
$$

Sustituyendo en la última ecuación el valor de $V_{4}$ previamente obtenido y juntando términos, se tiene

$$
C_{2}-C_{4}=V_{1}-V_{3}+i\left(-V_{1}-2 V_{2}-V_{3}\right)
$$

Dividiendo esta última expresión entre lo obtenido previamente para $C_{1}-C_{3}$, se tiene finalmente

$$
\left(\mathrm{C}_{2}-\mathrm{C}_{4}\right) /\left(\mathrm{C}_{1}-\mathrm{C}_{3}\right)=i
$$

lo cual demuestra que los segmentos dirigidos entre los centros de los cuadrados de los lados opuestos son perpendiculares y de igual longitud. Una consecuencia de la demostración aritmética es que las longitudes iguales y la perpendicularidad no dependen de los valores específicos de las V's, por lo que el teorema demostrado tiene gran generalidad. El cuadrilátero no necesita ser convexo. Algunos lados pueden tener longitud cero (pues en ningún momento hemos dividido entre ninguno de ellos) y como se verá más adelante, los cuadrados podrían estar hacia afuera o hacia adentro de la figura. Estas situaciones quedan ilustradas en las figuras 7 a 12 , en las cuales los lados del cuadrilátero son $A B, B C, C D, D A$ y los centros de los cuadrados apoyados sobre ellos son respectivamente $a b, b c, c d$ y $d a$.

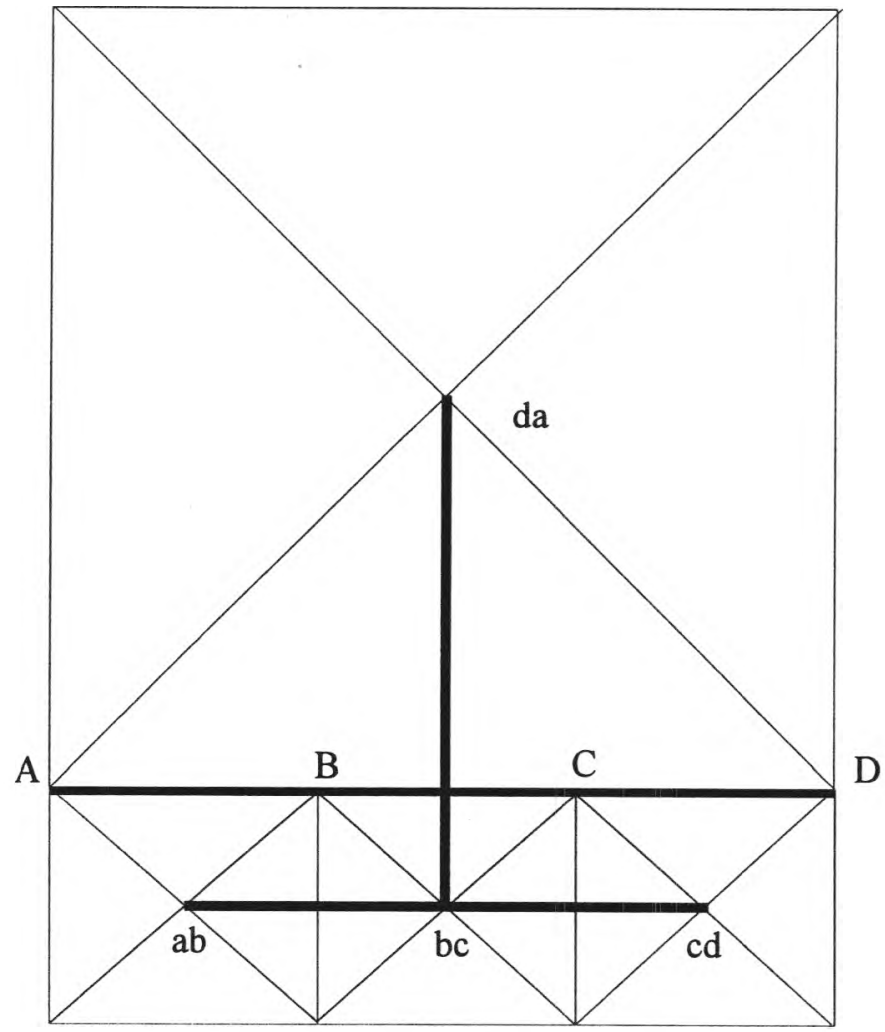

Figura 7. Los cuatro lados sobre una recta

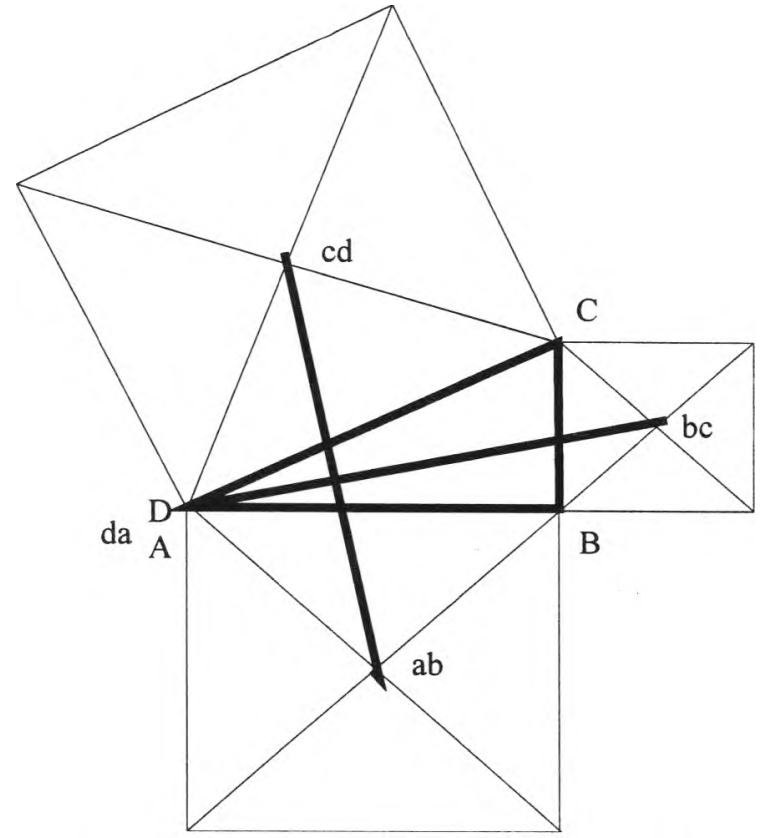

Figura 8. El lado DA tiene longitud 0

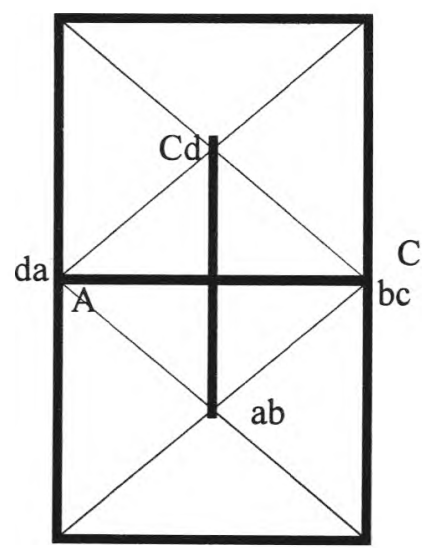

Figura 9.Los lados $B C$ y DA tienen longitud 0

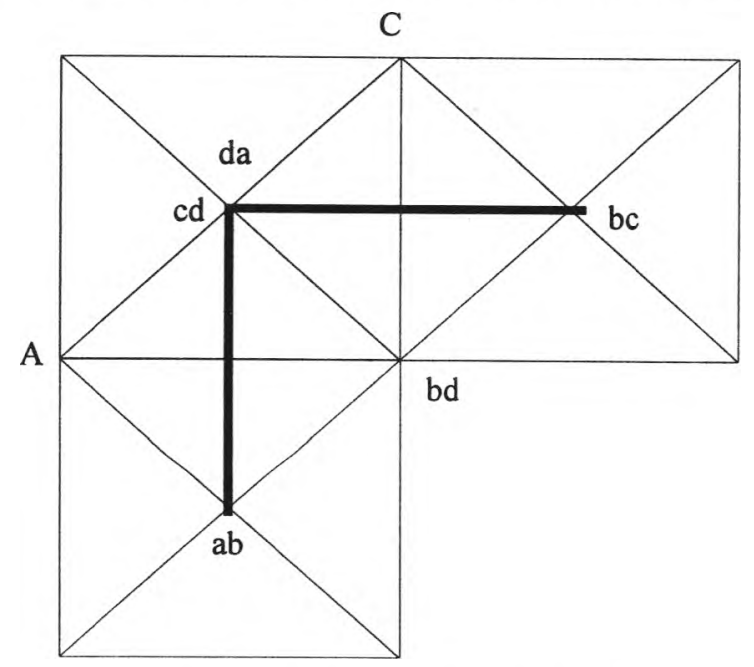

Figura 10. Cuadrilátero con área 0 , lados, no todos en una línea 


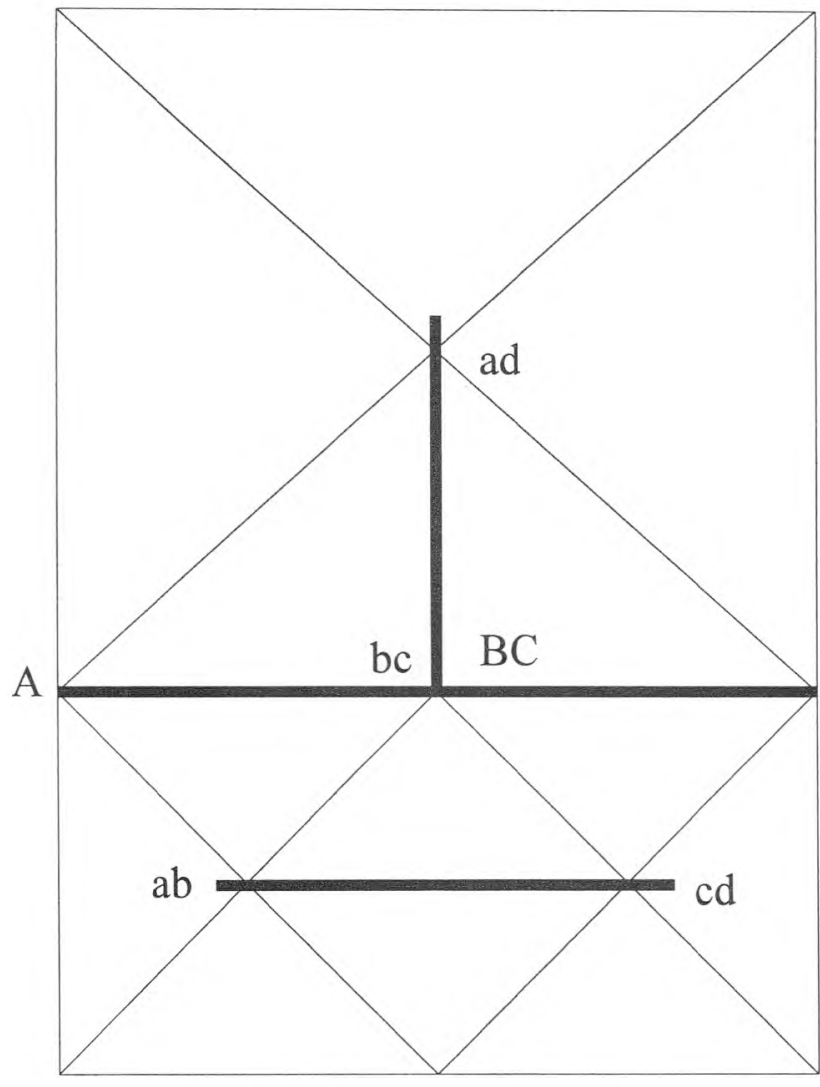

Figura 11. Cuadrilátero con área 0 , todos los vértices en una línea con un lado de longitud 0

\section{Demostración con números complejos del teorema de Desargues}

El teorema de Desargues es uno de los teoremas fundamentales de la geometría proyectiva (Courant y Robbins, 1969; Santaló, 1966 y Colerus, 1948) que tiene su contraparte en la geometría euclídea. Aquí se tratará como un teorema de la geometría euclídea plana y su demostración se hará usando números complejos. El teorema dice: si en un plano, dos triángulos $\mathrm{ABC}$ y A'B'C' están situados de tal manera que las líneas que unen los vértices correspondientes se encuentran en un punto $O$, entonces los lados correspondientes, si se prolongan, se cruzarán en tres puntos colineales (Figura 13).

D

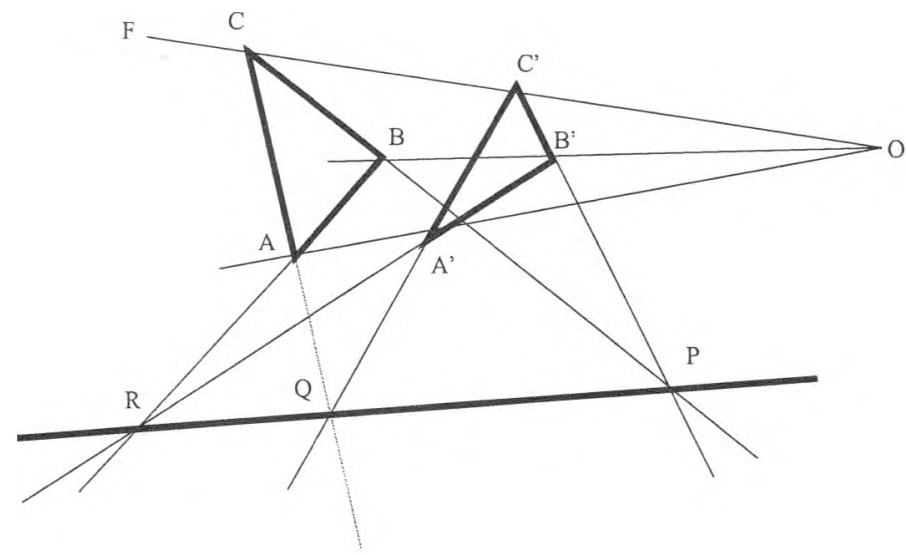

Figura 13

Para proceder a la demostración, escogeremos el origen del plano complejo en el punto $O$ de la Fig. 13. Llamaremos $A$, $B, C, A^{\prime}, B^{\prime}, C^{\prime}$ a los números complejos cuyas representaciones en el plano complejo son los segmentos de recta orientados que van del punto $\mathrm{O}$ a los puntos con los mismos nombres $\left(A, B, \ldots, C^{\prime}\right.$.) de la Fig. 13. Por estar los puntos $A$ y $A^{\prime}, B$ y $B^{\prime}$ y $C$ y $C^{\prime}$ en rayos que parten del origen se puede escribir

$$
A^{\prime}=a A, B^{\prime}=b B, C^{\prime}=c C
$$

donde $a, b, c$ son números reales. Si llamamos con $P, Q, R$ a los números complejos cuya representación en el plano complejo son los segmentos de recta orientados que van del punto $\mathrm{O}$ a los puntos $\mathrm{P}, \mathrm{Q}, \mathrm{R}$ de la Fig. 13, entonces debido a que dichos puntos están en el cruce de las prolongaciones de los lados $B C$ y $B^{\prime} C^{\prime} ; A C$ y $A^{\prime} C^{\prime}$; y $A B$ y $A^{\prime} B^{\prime}$ respectivamente, podremos escribir

$$
\begin{gathered}
P=B+k_{1}(C-B)=B^{\prime}+k_{1}{ }^{\prime}\left(C^{\prime}-B^{\prime}\right)= \\
b B+k_{1}{ }^{\prime}(c C-b B) \\
Q=C+k_{2}(C-A)=C^{\prime}+k_{2}^{\prime}\left(C^{\prime}-A^{\prime}\right)=
\end{gathered}
$$

Figura 12. Cuadrilátero con área 0 , vértices no todos en una linea 


$$
\begin{gathered}
c C+k_{2}^{\prime}(c C-a A) \\
R=A+k_{3}(A-B)=A^{\prime}+k_{3}^{\prime}\left(A^{\prime}-B^{\prime}\right)= \\
a A+k 3^{\prime}(a A-b B)
\end{gathered}
$$

donde $k_{1}, k_{2}, k_{3}, k_{1}{ }^{\prime}, k_{2}{ }^{\prime}, k_{3}{ }^{\prime}$ son números reales. Para que estos números sean independientes del conjunto $\alpha=\{$ $\left.A, B, C, A^{\prime}, B^{\prime}, C^{\prime}\right\}$, se igualan los coeficientes de los números complejos del conjunto $\alpha$, lo cual da 6 ecuaciones con las 6 incógnitas $k_{1}, k_{2}, k_{3}, k_{1}{ }^{\prime}, k_{2}{ }^{\prime}, k_{3}{ }^{\prime}$. Dichas ecuaciones están altamente desacopladas, por lo cual son fáciles de resolver. Por ejemplo, de la última ecuación igualando los coeficientes de $\mathrm{A}$ y $\mathrm{C}$ se obtienen las dos ecuaciones

$$
1+k_{3}=a\left(1+k_{3}{ }^{\prime}\right), k_{3}=k_{3}{ }^{\prime} b
$$

de donde se obtiene

$$
k_{3}{ }^{\prime}=(1-a) /(a-b)
$$

En forma similar se obtienen

$$
k_{1}{ }^{\prime}=(1-b) /(c-b), k_{2}{ }^{\prime}=(1-c) /(c-a)
$$

Utilizando estos valores podremos escribir después de colectar términos

$$
\begin{aligned}
& P=\mathrm{Cc}(1-b) /(c-b)+\mathrm{Bb}(c-1) /(c-b) \\
& \mathrm{Q}=\mathrm{Cc}(1-a) /(c-a)+\mathrm{A} a(c-1) /(c-a) \\
& \mathrm{R}=\mathrm{A} a(1-b) /(a-b)+\mathrm{Bb}(a-1) /(a-b)
\end{aligned}
$$

Los puntos $\mathrm{P}, \mathrm{Q}, \mathrm{R}$ serán colineales si el cociente $(\mathrm{R}$ $P) /(R-Q)$ es un número real. Esto debe suceder independientemente de los valores de los elementos del conjunto $\alpha$, exclusivamente bajo la suposición de que las tres líneas $\mathrm{AA}^{\prime}, \mathrm{BB}^{\prime}$ y CC' se encuentran en el mismo punto $\mathrm{O}$. Lo anterior se cumple si las razones entre los coeficientes de $A$, $B$ y $C$ de $(R-P)$ y $(R-Q)$ son iguales. Dicha igualdad se puede verificar haciendo las operaciones algebraicas correspondientes.

El coeficiente de A en la expresión de

$$
R-P \text { es: } a(1-b) /(a-b)
$$

El coeficiente de A en la expresión de

$$
R-Q \text { es }: a[(1-b) /(a-b)+(1-c) /(c-a)]
$$

La razón del primero al segundo es, tras un poco de álgebra:

$$
[(1-b)(c-a)] /[(1-a)(c-b)]
$$

El coeficiente de $B$ en la expresión de

$$
\mathrm{R}-\mathrm{P} \text { es: } b[(a-1) /(a-b)+(1-c) /(c-b)]
$$

El coeficiente de B en la expresión de

$$
R-Q \text { es: } b(a-1) /(a-b)
$$

La razón del primero al segundo es, tras un poco de álgebra:

$$
[(1-b)(c-a)] /[(1-a)(c-b)]
$$

El coeficiente de $\mathrm{C}$ en la expresión de

$$
R-P \text { es: } c(b-1) /(c-b)
$$

El coeficiente de $C$ en la expresión de

$$
R-Q \text { es: } c(1-a) /(a-c)
$$

La razón del primero al segundo es:

$$
[(1-b)(c-a)] /[(1-a)(c-b)]
$$

Como la razón es igual para los tres coeficientes y es independiente de $A, B \circ C$, hemos demostrado el teorema y este es válido con gran generalidad. Por ejemplo, no es necesario que los puntos A, B, Co los puntos A', B' C' estén del mismo lado del punto $O$, basta que se situen en las mismas rectas y que éstas pasen por $O$. En la figura 14 se muestra un ejemplo.

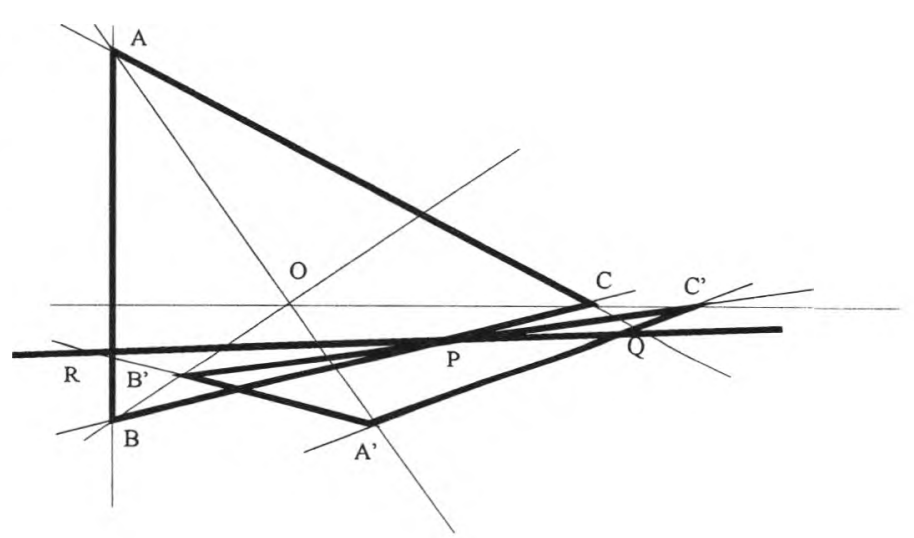


En la Fig. 14, dos de los puntos del primer triángulo $A B C$ están a la izquierda del punto $O$ y el tercer punto (C) está del otro lado. También, el triángulo $A^{\prime} B^{\prime} C^{\prime}$ tiene dos de sus vértices ( $\left.A^{\prime} C^{\prime}\right)$ de un lado del punto $O$ y el otro vértice ( $\left.B^{\prime}\right)$ en el otro lado. El teorema se cumple de todos modos y los puntos $P, Q, R$ son colineales.

\section{Conclusiones}

Debido a la posibilidad de representar un número complejo con un segmento de recta dirigido en el plano de Argand, los números complejos al igual que los vectores bidimensionales tienen la posibilidad de ser una herramienta muy útil en el estudio de la geometría plana. Ambos obedecen la ley de paralelogramo o ley del triángulo en la operación de sumar dos elementos, o la ley del polígono al sumar varios de ellos. Para los números complejos también se definen las operaciones de multiplicación y división. -Para los vectores bidimensionales está definido un producto especial llamado producto punto, producto escalar o producto interno. La división no está definida para vectores bidimensionales-

En este artículo se exhibieron varios casos de problemas y demostraciones de teoremas con el propósito de ilustrar las posibilidades del uso de números complejos en la enseñanza de la geometría plana. Una de las ventajas del uso de los números complejos es que las demostraciones tienen gran generalidad, la cual da el álgebra, que sin saber de antemano el signo, se presta para tomar el valor que convenga a una situación específica. Cuando las demostraciones de este tipo se hacen utilizando técnicas geométricas convencionales, es necesario tomar diversos casos particulares en consideración. Lo anterior se exhibió en este trabajo con el teorema de Von Aubel.

Dada la posible utilidad de los números complejos para la enseñanza de la geometría plana, el autor recomienda la elaboración de más material educativo sobre este tema. Ante todo, daría la oportunidad de introducir los números complejos más temprano en el currículum, dándoles una aplicación muy tangible que restaría la mistificación que se les ha otorgado tradicionalmente a los números complejos. Los números complejos tienen importantes aplicaciones en la ciencia y la tecnología. Son muy útiles en el estudio de la relatividad y la mecánica cuántica, así como en el estudio de la corriente alterna en electricidad y en el estudio de vibraciones mecánicas, pues con ellos, se pueden combinar senoides con diferentes fases de una manera muy simple. Son también introductorios de la teoría de funciones de variable compleja, la cual tiene gran importancia tanto matemática como física en el estudio de campos eléctricos, magnéticos y de otros tipos, mecánica de fluídos, mecánica de suelos, de sólidos, y muchas otras disciplinas, por lo que merecen una mayor presencia en los planes de estudio de la secundaria y preparatoria.

En el tipo de aplicaciones que se dieron en este artículo, los números complejos como las matrices y los vectores, permiten una gran economía de pensamiento. Así cualquier polígono cerrado, da pie a una ecuación entre números complejos que representan a los lados cuya suma (como número complejo) es cero. Es conveniente aclarar que todo lo que se mostró en este artículo, se puede realizar utilizando vectores bidimensionales, no obstante, el autor considera que puede ser conveniente tener más de un punto de vista en cualquier situación. Así por ejemplo, para sumar senoides de igual frecuencia, los ingenieros eléctricos prefieren representar cada senoide por medio de un número complejo que recibe el nombre de fasor y utilizar la aritmética de los números complejos para encontrar la suma de senoides. Hay personas que prefieren utilizar fórmulas trigonométricas para el mismo propósito. Sobre la misma línea, los ingenieros eléctricos utilizan funciones de variable compleja para manejar sistemas dinámicos invariantes en el tiempo. Otras personas prefieren utilizar las ecuaciones diferenciales directamente. Fueron los ingenieros eléctricos (el ingeniero Oliver Heaviside) los que introdujeron los métodos operacionales en la solución de ecuaciones diferenciales ordinarias y diferenciales parciales, las cuales se han justificado posteriormente de diversas maneras, una de ellas por medio de variable compleja y las transformadas de Laplace y de Fourier. La moraleja que quisiera dejar el autor es: "vale la pena explorar más de un punto de vista en la visualización y solución de problemas matemáticos."

\section{Referencias}

Colerus E. (1948). Desde el punto a la cuarta dimensión. Editorial Labor, SA. Barcelona.

Courant R. y Robbins H.(1969). What is Mathematics? Oxford University Press, Londres.

Gardner M. (1981). Mathematical Circus. Vintage Books. Random House, New York.

Kelly P.(1966).Von Aubel's Quadrilateral Theorem, Mathematics Magazine, enero. 35 - 37.

Klein F. (1939). Elementary Mathematics from an Advanced Standpoint-Geometry. Dover Publications, New York.

Santaló L.A. (1966). Geometría proyectiva. Editorial Universitaria de Buenos Aires, Buenos Aires.

Yaglom I.M. (1962). Geometric Transformations. New Mathematical Library, Random House, New York. 
DOI: http://dx.doi.org/10.22201/fi.25940732e.2000.01n5.023

\section{Semblanza del autor}

Marco Antonio Murray-Lasso. Realizó la licenciatura en ingeniería mecánica-eléctrica en la Facultad de Ingeniería de la UNAM. El Instituto de Tecnología de Massachussetts (MIT) le otorgó los grados de maestro en ciencias en ingeniería eléctrica y doctor en ciencias cibernéticas. En México, ha laborado como investigador en el Instituto de Ingeniería y como profesor en la Facultad de Ingeniería, UNAM durante 36 años; en el extranjero, ha sido asesor de la NASA en diseño de circuitos por computadora para aplicaciones espaciales, investigador en los Laboratorios Bell, así como profesor de la Universidad Case Western Reserve y Newak College of Engineering, en los Estados Unidos. Ha ocupado diversos puestos en sociedades profesionales relativas a la ingeniería,tanto nacionales como internacionales. Es coautor de libros y artículos técnicos, memorias de conferencias y revistas de investigación y divulgación. Actualmente, es jefe de la Unidad de Enseñanza Auxiliada por Computadora de la División de Estudios de Posgrado de la Facultad de Ingeniería, UNAM, Investigador Nacional-SNI y consultor de la UNESCO. 